\title{
Cognitive dysfunction in autoimmune rheumatic diseases
}

\author{
Csaba Oláh ${ }^{1 \dagger}$, Noa Schwartz ${ }^{2 \dagger}$, Christopher Denton ${ }^{3+}$, Zsófia Kardos ${ }^{4}$, Chaim Putterman ${ }^{2,5,6,7}$ and Zoltán Szekanecz ${ }^{8^{*}}$
}

\begin{abstract}
For people with chronic autoimmune rheumatic diseases (AIRD), such as rheumatoid arthritis (RA), systemic lupus erythematosus (SLE) or systemic sclerosis (SSC), normal cognitive functions are essential for performing daily activities. These diseases may be associated with cognitive dysfunction (CD). In RA, CD has been associated with age, lower education and disease duration and activity. Great advances have been achieved in neuropsychiatric SLE in the identification of pathogenic pathways, assessment and possible treatment strategies. SSc rarely exerts direct effects on the brain and cognitive function. However, the psychological burden that includes depression, anxiety and social impact may be high. AIRD patients with sustained disease activity, organ damage or lower education should be evaluated for CD. The control of systemic inflammation together with tailored behavioural cognitive therapies may benefit these patients.

Keywords: Cognitive function, Cognitive dysfunction, Rheumatoid arthritis, Systemic lupus erythematosus, Neuropsychiatric lupus, Systemic sclerosis
\end{abstract}

\section{Introduction}

Cognitive function includes orientation, attention/concentration, judgment/problem solving and memory, verbal, visual/spatial and executive functions [1]. Several standardised measures of cognitive function have been developed. These include the Montreal Cognitive Assessment (MoCA), Trail Making Test (TMT), Victoria Stroop Test (VST), Wechsler Adult Intelligence Scale (WAIS) and Benton Visual Retention Test (BVRT). Beck Depression Inventory (BDI) and State-Trait Anxiety Inventory (STAIT/S) may be used to assess depression and anxiety, respectively [2]. For people with chronic illnesses, such as autoimmune-inflammatory rheumatic diseases (AIRD), intact cognitive functioning is crucial for performing many key daily tasks, including medical treatment adherence or planning activities. Most AIRDs have been associated with various degrees of

\footnotetext{
* Correspondence: Szekanecz.zoltan@med.unideb.hu

${ }^{+}$Csaba Oláh, Noa Schwartz and Christopher Denton shared the first authorship with equal contribution.

${ }^{8}$ Division of Rheumatology, Faculty of Medicine, University of Debrecen, Nagyerdei str 98, Debrecen 4032, Hungary

Full list of author information is available at the end of the article
}

cognitive dysfunction (CD) (reviewed in $[1,3,4])$. In this review, we will briefly discuss $C D$ in rheumatoid arthritis (RA), systemic lupus erythematosus (SLE) and systemic sclerosis (SSc).

\section{Rheumatoid arthritis \\ Introduction}

RA exerts various neuropsychiatric manifestations [5]. There is an increased risk of stroke, a consequence of accelerated inflammatory atherosclerosis, especially in elderly RA patients with a long-standing disease [5]. Among psychiatric manifestations, depression and anxiety are present in two thirds of RA patients and have been associated with disease activity [6].

In RA, patients may significantly underperform on cognitive function tests compared to controls $[1,2,7]$. Even mild CD may influence the functional capacity and quality of life of these patients $[1,7,8]$. It affects reactivity to pain, compliance to and effectiveness of therapy [1]. This CD may occur early, in young RA patients, even in the early stages of the disease [9]. In general, CD 
may influence daily activities, treatment compliance and overall self-care $[1,2]$. Meade et al. [1] have recently performed the first systematic review of CD in RA.

\section{Pathogenesis}

Both inflammation itself that also affects the brain $[1,8]$ and accelerated atherosclerosis also driven by systemic inflammation including pro-inflammatory cytokines [10] may cause CD in RA. RA disease activity associated with systemic inflammation may be an important driver of $\mathrm{CD}$, as well as depression and anxiety $[1,8]$. Clinical features, such as pain, stress, fatigue and sleep disturbances, may be involved in the development of RA-associated CD. For example, depression can lead to abnormal concentration and executive function $[1,11]$. Among antirheumatic drugs, methotrexate (MTX) and corticosteroids have been associated with $\mathrm{CD}$. The effects of these compounds on RA-related CD may be controversial. Both MTX and corticosteroids dampen systemic inflammation and thus may have beneficial effects on cognitive function. On the other hand, MTX has been associated with $\mathrm{CD}$, mood changes and confusion, while corticosteroids may have an impact on memory and hippocampal function $[1,12]$.

\section{Assessment tools}

We have recently applied numerous standard validated measures in order to assess cognitive function, depression and anxiety in RA patients in association with the assessment of cerebral circulation and pathology by transcranial Doppler (TCD), carotid ultrasound and brain MRI [2]. Standard measures of CD including MoCA, TMT, VST, WAIS and BVRT, as well as BDI and STAI measuring depression and anxiety, showed impairment in RA vs controls. Biologic- vs MTX-treated subgroups also showed some difference in a few measures [2]. Shin et al. [7] used 16 cognitive indices and found a relationship between cognitive and functional limitations. We have reported the impairment of most mental domains in the SF36 quality of life assessment tool in RA [2]. In our study, numerous cognitive tests correlated with age and lower education. Some also correlated with disease duration, ESR and disease activity [2]. Shin et al. [7] and Lee et al. [13] also associated CD with low education and disease activity, respectively. Regarding vascular pathophysiology, cerebral vascular lesions, TCD parameters and carotid plaques were associated with impairment of multiple cognitive parameters [2].

\section{Management}

The control of disease activity may be essential in this respect $[1,8]$. Although preliminary studies suggested that MTX may aggravate CD, the beneficial effects of MTX and biologics on the suppression of disease activity may override this potential effect [1]. Although proinflammatory mediators have been implicated in the development of CD in RA [1, 8], only very few studies evaluated the longitudinal effects of anti-rheumatic drugs on cognitive function. In the open-label study of Raftery et al. [14], adalimumab improved full scale, verbal and performance IQ in parallel with improvements of DAS28. Camara et al. [15] reported that centrally administered etanercept improved anxiety-like behaviour in mice. In addition to pharmacotherapy, tailored cognitive-behavioural therapy (CBT) administered early in the course of RA also reduced health care use for the first 5 years after treatment [16].

\section{Systemic lupus erythematosus Introduction}

Neuropsychiatric manifestations in SLE, formerly known as lupus cerebritis, are diverse and common. An American College of Rheumatology (ACR) ad hoc committee in 1999 described 19 neuropsychiatric syndromes that can be directly attributed to SLE, ranging from stroke, acute confusional state and transverse myelitis to more low-grade, non-specific symptoms such as headaches, mood disorders and cognitive impairment [17]. While often considered subtle and non-specific, lupus patients have identified $C D$ as one of the most distressing symptoms of their disease. Studies have estimated that CD affects $3-81 \%$ of lupus patients [3], the wide range resulting from non-standardised diagnostic criteria and screening tools, and difficulty in the attribution of symptoms to SLE. Additional hurdles in the diagnosis of neuropsychiatric manifestations are that they often develop insidiously, can present and progress independently of other signs of SLE activity and often do not respond to standard immunosuppression. Thus, there is growing recognition that $\mathrm{CD}$ in lupus patients remains underdiagnosed and under-addressed in the clinical setting [18].

\section{Pathogenesis}

Suffering from a chronic disease state, taking neurotoxic/ psychoactive medications such as corticosteroids and cyclophosphamide, and other manifestations of neuropsychiatric SLE (NPSLE) such as strokes, seizures, depression or anxiety can all independently lead to $\mathrm{CD}$. Nevertheless, the early presentation of neuropsychiatric symptoms $(40 \%$ of lupus patients suffer from neuropsychiatric symptoms on presentation or within the first 3 years of disease diagnosis $[19,20])$, the increased prevalence of CD in lupus patients compared to other chronic inflammatory diseases [3], in addition to animal models displaying associated behaviours [21], strongly points to a disease-specific, immunologic aetiology. Pathogenic pathways thought to be instrumental in NPSLE have been comprehensively described elsewhere [21], but here, we will briefly discuss those that have been specifically linked to 
cognitive dysfunction. Antiphospholipid (aPL) antibodies, often associated with SLE and known to be a strong risk factor for NPSLE, lead to a hypercoagulable state often resulting in strokes, structural damage and associated CD [21, 22]. Of note, aPL antibodies have also been linked to less clearly understood NPSLE syndromes that are not necessarily directly related to thrombosis or ischaemic events [23]. In fact, there is evidence for the involvement of multiple aspects of the immune system in NPSLE, including neurotoxic autoantibodies, pro-inflammatory cytokines and cell-mediated effects, in conjunction with abnormalities in neuroimmune interfaces including the choroid plexus and blood-brain barrier which allow systemic autoimmune drivers into the central nervous system [21, 24]. Specifically, several studies have pointed to increased systemic levels of cytokines such as interleukin 6 (IL-6) and neurotoxic anti- $N$-methyl-D-aspartate receptor (NMDAR) antibodies in SLE patients with CD [25-27]. In addition, type I interferon (IFN-I) and anti-NMDAR antibodies were shown to enhance microglia activation, leading to aberrant synaptic pruning with subsequent $C D[28,29]$. There is some experimental evidence to suggest that microglia activation can be dampened with both antiIFN- $\alpha / \beta$ receptor (IFNAR) treatment and the bloodbrain barrier-crossing angiotensin-converting enzyme (ACE) inhibitor captopril. These interventions were associated with improvement in neurobehavioural deficits in lupus mice $[28,29]$, although there was a conflicting report with anti-IFNAR treatment and its effect on neuropsychiatric symptoms [30].

While promising directions in terms of investigations into the pathogenesis, as well as specific treatments of $\mathrm{CD}$, are starting to accrue, those are still mainly focused on animal disease models, limiting our ability to extrapolate these findings to human disease [21, 28-30].

\section{Measurement tools}

The ACR 1999 nomenclature defined CD as including a significant deficit in any or all of the following cognitive domains: simple or complex attention, reasoning, executive skills, memory, visual-spatial processing, language and psychomotor speed [17]. Among these, in SLE, language and working memory domains are most commonly affected, followed by simple attention and psychomotor speed [3]. Rayes et al. [3] presented a comprehensive meta-analysis evaluating different neurocognitive testing tools used to assess CD in SLE. While the 4-h, complete battery of neurocognitive testing is the most comprehensive, it is not feasible for routine assessments of patients in the clinical setting. A number of alternative modalities have been utilised with varying degrees of agreements with the complete neurocognitive testing battery, including the ACR-SLE battery [17], a 1$\mathrm{h}$ battery of tests that focus on SLE-specific domains that show good agreement with the complete battery in healthy controls and SLE patients without NPSLE (96\% and $95 \%$ agreement, respectively, with 100\% sensitivity in both groups, and 96\% specificity in controls and $94 \%$ in SLE without NPSLE), but only moderate agreement in patients with NPSLE (81\% agreement, with $80 \%$ sensitivity and $81 \%$ specificity) [31]. While the ACR-SLE battery has been shown to be a relatively good $C D$ evaluating tool in SLE patients, its main barrier is the significant time and resources it requires for administration, making it challenging for routine use. The Automated Neuropsychological Assessment Metric (ANAM) is a 40-min, self-administered computerised test that has been validated in multi-ethnic populations and is less influenced by age, education, English language proficiency and depression than other forms of neurocognitive testing [32, 33]. When compared to the ACR-SLE battery, the ANAM was found to have a sensitivity of $78-80 \%$ and specificity of $70 \%$ [32, 33], demonstrating promise as a possible CD screening method. The ANAM, however, needs further analysis of its capacity to monitor changes in $\mathrm{CD}$ over time and is thought to lack the ability to identify specific domain impairments [3]. Finally, the MoCA test, already mentioned above, is a brief, 15min administered test that showed acceptable agreement with the ANAM (sensitivity $83 \%$ and specificity $73 \%$ ) but requires more extensive validation prior to clinical and investigational use [34]. Identifying and validating a reliable screening and monitoring tool for $\mathrm{CD}$ that is both sensitive and specific, as well as technically feasible in the clinical setting, are of the utmost importance. Relying on patients' self-reporting is clearly inadequate, especially as studies comparing patient self-reporting to objective findings of $\mathrm{CD}$ found no association between the two, most probably because self-perception of $\mathrm{CD}$ is often closely associated with depression and anxiety [35]. It is also important to emphasise that these tests may be useful for the identification and quantification of $\mathrm{CD}$, but are not specific for NPSLE, and do not allow the discerning of primary SLE-related CD from any other confounding causes.

\section{Advanced imaging studies}

The pathways underlying lupus $\mathrm{CD}$ are still being explored, and the currently available diagnostic tools are not specific and/or have yet to be fully validated and broadly accepted. Thus, there remains a need for an objective and non-invasive means to establish neurological dysfunction in SLE patients both for investigative purposes, as well as for clinical diagnosis and surveillance. There is evidence to suggest that SLE patients, with and without overt NPSLE, have aberrations in their white 
matter microstructures as seen by diffusion tensor imaging (DTI) [36]. Functional MRI studies have shown increased functional connectivity in core resting-state networks in SLE patients that correlate with reduced cognitive performance compared with healthy controls [37]. FDG-PET imaging highlighted hypermetabolic regions that corresponded with increased serum antiNMDAR antibodies in SLE patients with CD and did not correlate with disease duration, activity, medications or comorbidities $[26,27]$. These studies highlight a potential role for these imaging techniques in objectively diagnosing and monitoring CD in SLE patients.

\section{Attribution of CD to SLE}

The potential use of imaging studies to diagnose CD in SLE in an unbiased and disease-specific setting is appealing, especially as there are numerous confounders that can affect CD in SLE patients. As we noted, mood disorders, depression, chronic pain, corticosteroid use and struggling with a chronic illness from a young age are all known to contribute to $\mathrm{CD}$ and are quite common in SLE patients. In order to better study, address and manage CD symptoms in SLE patients, it is important to first be able to correctly attribute them to the disease itself. To that end, several models have been devised to aid clinicians in attributing the symptoms to SLE (comprehensively reviewed in [38]). These models take into account factors such as the temporal relationship between the onset of neuropsychiatric symptoms and diagnosis or change in the activity of SLE, viable alternative explanations and the presence of risk factors specific to SLE such as aPL antibodies [22]. However, these models have been developed with the expert opinion being the diagnostic gold standard, with no objective corroborating evidence. Therefore, the attribution of CD to SLE remains mainly reliant on the clinician's assessment, index of suspicion and clinical reasoning, which can benefit from the introduction of a collaborative multidisciplinary approach including neuropsychological, radiological and laboratory evaluations $[21,39]$.

\section{Systemic sclerosis Introduction}

Scleroderma encompasses several significant immunemediated diseases that lead to thickening or fibrosis of the skin. The most severe forms medically are those in which systemic involvement occurs, namely SSc [40]. It has been suggested that the central nervous system is one of the very few organs that is not involved in SSc, but in fact, there are several aspects of the disease where cognitive function is clearly relevant and important. The localised forms of scleroderma, termed morphoea, are also important in terms of functional and cosmetic burden due to their impact on local connective tissue growth and appearance. Interestingly emotional stress is recognised as a factor in the development of scleroderma, and it is not uncommon for patients to describe major emotional difficulties such as bereavement or divorce in the 12-18 months prior to disease onset [41]. In this way, the interplay between the neurocognitive processes and immune or inflammatory disease is highlighted.

\section{Psychological burden in SSC}

More specific issues occur due to the impact of SSc on physical appearance and function. This can impact on almost all aspects of daily life and has a major detrimental effect on social interaction. Reasons for this include the effect on appearance as well as difficulties related to eating, working or participating in normal sociable activities. The impact is clear from studies that have used validated instruments to assess burden [42]. In addition, there has been a clear demonstration that clinical interventions that can improve facial appearance, such as autologous fat transfer, can greatly improve the score of psychological well-being [43].

There have been recent attempts to better describe and quantify psychological burden in SSc, and these have used the randomised cohort design of the SPIN project [44]. This approach allows greater evidence gathering to assess the utility of measurement tools as well as possible intervention strategies [45]. This is important because the psychological impact of SSc is frequently highlighted by patients as a major unmet need and one that is not well addressed in current approaches to treatment $[44,45]$. The shared aspects across other immunemediated or chronic diseases may be important in planning future interventions.

\section{Cognitive impairment in SSC}

In general, although anxiety, depression and mood difficulties are common, CD in SSc is not a frequent complication of the disease process. This contrasts with other AIRDs and might reflect fundamental differences in the cytokine or other immune cell drives across diseases [46]. There are however instances where vascular damage can be relevant. The most striking example is in the context of scleroderma renal crisis. This is a form of thrombotic microangiopathy associated with accelerated phase hypertension that occurs almost exclusively in early-stage diffuse cutaneous SSc and is strongly associated with the anti-RNA polymerase autoantibodies. There is a propensity to end-organ damage that may reflect the systemic microvasculopathy of SSc and may lead to generalised seizures and on occasion significant CD [47]. Fortunately, this generally recovers over several weeks without sequelae. A recent study formally assessed cognitive function in a cohort of SSc cases found evidence of cognitive impairment compared with matched healthy controls and 
that this associated with worse vascular disease. Future studies may explore whether this relates to comorbidity or is a specific feature of SSc [48].

Approximately one in five patients with SSc will also manifest additional features of another AIRD, such as SLE or Sjögren's syndrome [49]. This is important because the cognitive impact of these other diseases will be an additional burden for patients and may sometimes be the predominant problem, as outlined in other parts of this article. Immune-mediated encephalitic states may occur occasionally in this context [50].

Finally, it is notable that SSc is associated with Raynaud's phenomenon, and there have been several reports of mental or cognitive impact of cold exposure [4]. This has in some cases been associated with changes in cerebral perfusion scan and raises the intriguing possibility that Raynaud's might on occasion affect the cerebral circulation [4]. This has not been definitely shown, and more research is needed. Likewise, in localised scleroderma, especially linear morphoea, there can be structural abnormalities affecting the cranium or brain substance. This has been associated with epilepsy and possibly also with cognitive complications in some cases.

\section{Conclusion}

Patients with RA, SLE or SSc may exert significant cognitive impairment. In RA, various domains of cognitive dysfunction may be associated with age, lower education, disease duration and activity, inflammatory markers and some measures of vascular pathology. Important strides have been made in NPSLE- and SLE-specific CD in particular, in the elucidation of the pathogenic pathways and possible treatment strategies, imaging studies, and the design and validation of clinically feasible and diagnostically acceptable screening and monitoring tools. SSc rarely directly affects brain function, but the psychological burden can be large including depression anxiety and social impact. This represents a major unmet need that will likely remain a key problem despite promising data from ongoing and recent clinical trials of potential therapies for skin or lung manifestations of the disease. Although there have been fewer studies of cognitive impairment in SSc that for SLE or RA, it is important and maybe addressed in future work, perhaps utilising the randomised cohort design of the SPIN consortium [44]. From the practical point of view, AIRD patients, especially those with higher age, lower education, sustained disease activity and higher damage indices, should be evaluated for $C D$ by any standard test. Although some anti-rheumatic agents may impair cognitive function, the control of inflammation by conventional and biologic DMARDs, in association with tailored behavioural cognitive therapies, may benefit our AIRD patients. There obviously is still much work to be done in developing the right tools to best serve and optimally manage these patients with this distressing complication of an already challenging disease.

\section{Acknowledgements \\ There is nobody to acknowledge who contributed towards the article who does not meet the criteria for authorship. Funding details are included above.}

\section{Availability of supporting data}

N/A

\section{Authors' contributions}

CO performed the literature review for the "Rheumatoid arthritis" section, drafted the manuscript and assessed the final manuscript. NS performed the literature review for the "Systemic lupus erythematosus" section, drafted the manuscript and assessed the final manuscript. CD performed the literature review for the "Systemic sclerosis" section, drafted the manuscript, assessed the final manuscript and participated in the concept design. ZK performed the literature review for the "Rheumatoid arthritis" section, drafted the manuscript and assessed the final manuscript. CP performed the literature review for the "Systemic lupus erythematosus" section, drafted the manuscript, assessed the final manuscript and participated in the concept design. ZS performed the literature review for the "Rheumatoid arthritis" section, drafted the manuscript, assessed the final manuscript, participated in the concept design and put together the sections in the draft. All authors read and approved the final manuscript.

\section{Funding}

This research was supported by the European Union and the State of Hungary and co-financed by the European Social Fund in the framework of TAMOP-4.2.4.A/2-11/1-2012-0001 "National Excellence Program" (ZS), by the European Union grants GINOP-2.3.2-15-2016-00015 and GINOP-2.3.2-15-201600050 (ZS), by the Royal Free Charity Fund 97 and Scleroderma and Raynaud's UK (CD) and by the US National Institute of Arthritis and Musculoskeletal Diseases (R01 AR065594) (CP).

This funding was used to collect and interpret the information included in the review.

Availability of data and materials

This is a review. There are no data in this manuscript; therefore, this section is not applicable.

Ethics approval and consent to participate

Not needed as it is a review; no human data and human tissue were used.

Consent for publication

Not needed as no individual patients' data are presented.

\section{Competing interests}

The authors declare that they have no competing interests.

\section{Author details}

${ }^{1}$ Departments of Neurosurgery, Borsod County Teaching Hospital, Miskolc, Hungary. ${ }^{2}$ Division of Rheumatology, Albert Einstein College of Medicine, Bronx, NY 10461, USA. ${ }^{3}$ Centre for Rheumatology, Royal Free Campus, University College London, London, UK. ${ }^{4}$ Departments of Rheumatology, Borsod County Teaching Hospital, Miskolc, Hungary. ${ }^{5}$ Department of Microbiology \& Immunology, Albert Einstein College of Medicine, Bronx, NY 10461, USA. ${ }^{6}$ Azrieli Faculty of Medicine of Bar Ilan University, Zefat, Israel. ${ }^{7}$ Research Institute, Galilee Medical Center, Nahariya, Israel. ${ }^{8}$ Division of Rheumatology, Faculty of Medicine, University of Debrecen, Nagyerdei str 98, Debrecen 4032, Hungary. 


\section{Received: 11 December 2019 Accepted: 1 April 2020}

\section{Published online: 15 April 2020}

\section{References}

1. Meade T, Manolios N, Cumming SR, Conaghan PG, Katz P. Cognitive impairment in rheumatoid arthritis: a systematic review. Arthritis Care Res (Hoboken). 2018;70(1):39-52.

2. Olah C, Kardos Z, Andrejkovics M, Szarka E, Hodosi K, Domjan A, Sepsi M, Sas A, Kostyal L, Fazekas K, et al. Assessment of cognitive function in female rheumatoid arthritis patients: associations with cerebrovascular pathology, depression and anxiety. Rheumatol Int. 2020;40(4):529-40.

3. Rayes HA, Tani C, Kwan A, Marzouk S, Colosimo K, Medina-Rosas J, Mustafa A, Su J, Lambiris P, Mosca M, et al. What is the prevalence of cognitive impairment in lupus and which instruments are used to measure it? A systematic review and meta-analysis. Semin Arthritis Rheum. 2018;48(2):240-55.

4. Giuliodori G, Fraticelli P, Bartolini M, Cagnetti C, Baruffaldi R, Rocchi MB, Provinciali L, Gabrielli A, Silvestrini M. Cognitive and cerebral hemodynamic impairment in scleroderma patients. Eur J Neurol. 2009;16(12):1285-90.

5. Joaquim AF, Appenzeller S. Neuropsychiatric manifestations in rheumatoid arthritis. Autoimmun Rev. 2015:14(12):1116-22.

6. Fiest KM, Hitchon CA, Bernstein CN, Peschken CA, Walker JR, Graff LA, Zarychanski R, Abou-Setta A, Patten SB, Sareen J, et al. Systematic review and meta-analysis of interventions for depression and anxiety in persons with rheumatoid arthritis. J Clin Rheumatol. 2017;23(8):425-34.

7. Shin SY, Katz P, Wallhagen M, Julian L. Cognitive impairment in persons with rheumatoid arthritis. Arthritis Care Res (Hoboken). 2012;64(8):1144-50

8. Gorelick PB. Role of inflammation in cognitive impairment: results of observational epidemiological studies and clinical trials. Ann N Y Acad Sci. 2010;1207:155-62

9. Simos P, Ktistaki G, Dimitraki G, Papastefanakis E, Kougkas N, Fanouriakis A, Gergianaki I, Bertsias G, Sidiropoulos P, Karademas EC. Cognitive deficits early in the course of rheumatoid arthritis. J Clin Exp Neuropsychol. 2016; 38(7):820-9.

10. Szekanecz Z, Kerekes G, Der H, Sandor Z, Szabo Z, Vegvari A, Simkovics E, Soos L, Szentpetery A, Besenyei T, et al. Accelerated atherosclerosis in rheumatoid arthritis. Ann N Y Acad Sci. 2007;1108:349-58.

11. Nerurkar L, Siebert S, McInnes IB, Cavanagh J. Rheumatoid arthritis and depression: an inflammatory perspective. Lancet Psychiatry. 2019;6(2):16473.

12. Coluccia D, Wolf OT, Kollias S, Roozendaal B, Forster A, de Quervain DJ. Glucocorticoid therapy-induced memory deficits: acute versus chronic effects. J Neurosci. 2008:28(13):3474-8.

13. Lee $\mathrm{JH}$, Kim GT, Kim YK, Lee SG. Cognitive function of patients with rheumatoid arthritis is associated with disease activity but not carotid atherosclerotic changes. Clin Exp Rheumatol. 2018;36(5):856-61.

14. Raftery G, He J, Pearce R, Birchall D, Newton JL, Blamire AM, Isaacs JD. Disease activity and cognition in rheumatoid arthritis: an open label pilot study. Arthritis Res Ther. 2012;14(6):R263.

15. Camara ML, Corrigan F, Jaehne EJ, Jawahar MC, Anscomb H, Baune BT. Effects of centrally administered etanercept on behavior, microglia, and astrocytes in mice following a peripheral immune challenge. Neuropsychopharmacology. 2015;40(2):502-12.

16. Sharpe L, Allard S, Sensky T. Five-year followup of a cognitive-behavioral intervention for patients with recently-diagnosed rheumatoid arthritis: effects on health care utilization. Arthritis Rheum. 2008:59(3):311-6.

17. The American College of Rheumatology nomenclature and case definitions for neuropsychiatric lupus syndromes. Arthritis Rheum. 1999;42(4):599-608. [No authors listed]

18. Kello N, Anderson E, Diamond B. Cognitive dysfunction in systemic lupus erythematosus: a case for initiating trials. Arthritis Rheumatol. 2019;71(9): 1413-25.

19. Hanly JG, Urowitz MB, Su L, Bae SC, Gordon C, Wallace DJ, Clarke A, Bernatsky $\mathrm{S}$, Isenberg D, Rahman A, et al. Prospective analysis of neuropsychiatric events in an international disease inception cohort of patients with systemic lupus erythematosus. Ann Rheum Dis. 2010;69(3): 529-35

20. Steup-Beekman GM, Zirkzee EJ, Cohen D, Gahrmann BM, Emmer BJ, Steens SC, Bollen EL, van Buchem MA, Huizinga TW. Neuropsychiatric manifestations in patients with systemic lupus erythematosus: epidemiology and radiology pointing to an immune-mediated cause. Ann Rheum Dis. 2013;72(Suppl 2):ii76-9.
21. Schwartz N, Stock AD, Putterman C. Neuropsychiatric lupus: new mechanistic insights and future treatment directions. Nat Rev Rheumatol. 2019;15(3):137-52.

22. Bertsias GK, loannidis JP, Aringer M, Bollen E, Bombardieri S, Bruce IN Cervera R, Dalakas M, Doria A, Hanly JG, et al. EULAR recommendations for the management of systemic lupus erythematosus with neuropsychiatric manifestations: report of a task force of the EULAR standing committee for clinical affairs. Ann Rheum Dis. 2010;69(12):2074-82.

23. Brey RL, Gharavi AE, Lockshin MD. Neurologic complications of antiphospholipid antibodies. Rheum Dis Clin N Am. 1993;19(4):833-50.

24. Stock AD, Gelb S, Pasternak O, Ben-Zvi A, Putterman C. The blood brain barrier and neuropsychiatric lupus: new perspectives in light of advances in understanding the neuroimmune interface. Autoimmun Rev. 2017;16(6): 612-9.

25. Barraclough M, McKie S, Parker B, Jackson A, Pemberton P, Elliott R, Bruce IN. Altered cognitive function in systemic lupus erythematosus and associations with inflammation and functional and structural brain changes. Ann Rheum Dis. 2019;78(7):934-40

26. Ploran E, Tang C, Mackay M, Small M, Anderson E, Storbeck J, Bascetta B, Kang S, Aranow C, Sartori C, et al. Assessing cognitive impairment in SLE: examining relationships between resting glucose metabolism and antiNMDAR antibodies with navigational performance. Lupus Sci Med. 2019; 6(1):e000327.

27. Mackay M, Vo A, Tang CC, Small M, Anderson EW, Ploran EJ, Storbeck J, Bascetta B, Kang S, Aranow C, et al. Metabolic and microstructural alterations in the SLE brain correlate with cognitive impairment. JCl insight. 2019;4(1). https://doi.org/10.1172/jci.insight.124002. [Epub ahead of print].

28. Bialas AR, Presumey J, Das A, van der Poel CE, Lapchak PH, Mesin L, Victora G, Tsokos GC, Mawrin C, Herbst R, et al. Microglia-dependent synapse loss in type I interferon-mediated lupus. Nature. 2017;546(7659):539-43.

29. Nestor J, Arinuma Y, Huerta TS, Kowal C, Nasiri E, Kello N, Fujieda Y, Bialas A, Hammond T, Sriram U, et al. Lupus antibodies induce behavioral changes mediated by microglia and blocked by ACE inhibitors. J Exp Med. 2018; 215(10):2554-66

30. Huang MW, Stock AD, Mike EV, Herlitz L, Kolbeck R, Putterman C. Anti-IFNAR treatment does not reverse neuropsychiatric disease in MRL/lpr lupus mice. Lupus. 2019;28(13):1510-23.

31. Kozora E, Ellison MC, West S. Reliability and validity of the proposed American College of Rheumatology neuropsychological battery for systemic lupus erythematosus. Arthritis Rheum. 2004;51(5):810-8.

32. Reeves DL, Winter KP, Bleiberg J, Kane RL. ANAM genogram: historical perspectives, description, and current endeavors. Arch Clin Neuropsychol. 2007;22(Suppl 1):S15-37.

33. Holliday SL, Navarrete MG, Hermosillo-Romo D, Valdez CR, Saklad AR, Escalante A, Brey RL. Validating a computerized neuropsychological test battery for mixed ethnic lupus patients. Lupus. 2003;12(9):697-703.

34. Adhikari T, Piatti A, Luggen M. Cognitive dysfunction in SLE: development of a screening tool. Lupus. 2011;20(11):1142-6.

35. Hanly JG, Su L, Omisade A, Farewell VT, Fisk JD. Screening for cognitive impairment in systemic lupus erythematosus. J Rheumatol. 2012;39(7):1371-7.

36. Kozora E, Filley CM, Erkan D, Ulug AM, Vo A, Ramon G, Burleson A, Zimmerman RD, Lockshin MD. Longitudinal evaluation of diffusion tensor imaging and cognition in systemic lupus erythematosus. Lupus. 2018;27(11): 1810-8.

37. Nystedt J, Mannfolk $P$, Jonsen A, Nilsson P, Strandberg TO, Sundgren PC. Functional connectivity changes in core resting state networks are associated with cognitive performance in systemic lupus erythematosus. J Comp Neurol. 2019;527(11):1837-56.

38. Bortoluzzi A, Scire CA, Govoni M. Attribution of neuropsychiatric manifestations to systemic lupus erythematosus. Front Med. 2018:5:68

39. Magro-Checa C, Zirkzee EJ, Beaart-van de Voorde $\sqcup J$, Middelkoop HA, van der Wee NJ, Huisman MV, Eikenboom J, Kruyt ND, van Buchem MA, Huizinga TWJ, et al. Value of multidisciplinary reassessment in attribution of neuropsychiatric events to systemic lupus erythematosus: prospective data from the Leiden NPSLE cohort. Rheumatology (Oxford). 2017;56(10):1676-83.

40. Denton CP, Khanna D. Systemic sclerosis. Lancet. 2017:390(10103):1685-99.

41. Stojanovich L, Marisavljevich D. Stress as a trigger of autoimmune disease. Autoimmun Rev. 2008;7(3):209-13.

42. Mills SD, Kwakkenbos L, Carrier ME, Gholizadeh S, Fox RS, Jewett LR, Gottesman K, Roesch SC, Thombs BD, Malcarne VL, et al Validation of the social appearance anxiety scale in patients with systemic sclerosis: a 
scleroderma patient-centered intervention network cohort study. Arthritis Care Res (Hoboken). 2018;70(10):1557-62.

43. Almadori A, Griffin M, Ryan CM, Hunt DF, Hansen E, Kumar R, Abraham DJ, Denton CP, Butler PEM. Stem cell enriched lipotransfer reverses the effects of fibrosis in systemic sclerosis. PLoS One. 2019;14(7):e0218068.

44. Kwakkenbos L, Jewett LR, Baron M, Bartlett SJ, Furst D, Gottesman K, Khanna D, Malcarne VL, Mayes MD, Mouthon L, et al. The Scleroderma Patient-centered Intervention Network (SPIN) Cohort: protocol for a cohort multiple randomised controlled trial (cmRCT) design to support trials of psychosocial and rehabilitation interventions in a rare disease context. BMJ Open. 2013;3(8). https:/doi.org/10.1136/bmjopen-2013-003563.

45. Fox RS, Kwakkenbos L, Carrier ME, Mills SD, Gholizadeh S, Jewett LR, Roesch SC, Merz EL, Assassi S, Furst DE, et al. Reliability and validity of three versions of the brief fear of negative evaluation scale in patients with systemic sclerosis: a scleroderma patient-centered intervention network cohort study. Arthritis Care Res (Hoboken). 2018;70(11):1646-52.

46. Mouthon L, Alami S, Boisard AS, Chaigne B, Hachulla E, Poiraudeau S. Patients' views and needs about systemic sclerosis and its management: a qualitative interview study. BMC Musculoskelet Disord. 2017;18(1):230.

47. Penn H, Howie AJ, Kingdon EJ, Bunn CC, Stratton RJ, Black CM, Burns A, Denton CP. Scleroderma renal crisis: patient characteristics and long-term outcomes. QJM. 2007;100(8):485-94.

48. Khedr EM, El Fetoh NA, Gamal RM, Elzohri MH, Azoz NMA, Furst DE. Evaluation of cognitive function in systemic sclerosis patients: a pilot study. Clin Rheumatol. 2020. https://doi.org/10.1007/s10067-019-04884-9. [Epub ahead of print].

49. Pakozdi A, Nihtyanova S, Moinzadeh P, Ong VH, Black CM, Denton CP. Clinical and serological hallmarks of systemic sclerosis overlap syndromes. J Rheumatol. 2011;38(11):2406-9.

50. De Stefano P, Chizzolini C, Lalive PH, Lascano AM. Limbic encephalitis associated with systemic sclerosis. Mult Scler Relat Disord. 2018;24:142-4.

\section{Publisher's Note}

Springer Nature remains neutral with regard to jurisdictional claims in published maps and institutional affiliations. 